\title{
Perfil de enfermeiros em unidades de terapia intensiva: fatores de risco para doenças crônicas não transmissíveis
}

\author{
Nurses profile in intensive care units: risk factors for chronic non-communicable diseases \\ Perfil de enfermeras en unidades de cuidados intensivos: factores de riesgo de enfermedades \\ crónicas no transmisibles
}

Recebido: 24/06/2021 | Revisado: 30/06/2021 | Aceito: 02/08/2021 | Publicado: 07/08/2021

\author{
Maíza Radely Pereira Ferreira \\ ORCID: https://orcid.org/0000-0003-2120-5251 \\ Universidade Estadual de Ciências da Saúde de Alagoas, Brasil \\ E-mail: radelyferreira@hotmail.com \\ Bernardo do Rego Belmonte \\ ORCID: https://orcid.org/0000-0001-5225-5417 \\ Faculdade de Comunicação, Tecnologia e Turismo de Olinda, Brasil \\ E-mail: professorbernardobelmonte@gmail.com \\ Géssyca Cavalcante de Melo \\ ORCID: https://orcid.org/0000-0002-6774-857X \\ Universidade Estadual de Ciências da Saúde de Alagoas, Brasil \\ E-mail: gessycamelo@hotmail.com \\ Geórgia Maria Ricardo Félix dos Santos \\ ORCID: https://orcid.org/0000-0003-0154-597X \\ Universidade Estadual de Ciências da Saúde de Alagoas, Brasil \\ E-mail: georgia_felix@hotmail.com
}

\begin{abstract}
Resumo
Objetivo: Descrever a ocorrência de doenças crônicas não transmissíveis (DCNT) autorreferidas e os fatores de risco relacionados em enfermeiros da Unidade de Terapia Intensiva (UTI) Geral e Pediátrica em um hospital de referência na cidade de Maceió-AL. Métodos: Estudo descritivo, realizado no período de abril a julho de 2019. Os dados foram obtidos através de um questionário elaborado pelos autores, com perguntas referentes aos dados pessoais e sociodemográficos, hábitos ocupacionais e de vida e se já possuíam tais hábitos antes da atuação na UTI, além de dados antropométricos autorreferidos. Resultados: Foram entrevistados 16 enfermeiros, 87,5\% do sexo feminino, com idade entre 25 a 50 anos, que atuavam no setor entre 11 a 15 anos. Em relação ao Índice de Massa Corporal, 37,5\% apresentaram sobrepeso e 6,25\%, obesidade. Declararam ser fumantes $12,5 \%$ dos enfermeiros e $43,75 \%$ dos entrevistados relataram haver adquirido alguma DCNT ao longo da atuação profissional na UTI. Conclusão: A ocorrência de DCNT identificada e de fatores de risco para o seu desenvolvimento apresentou-se relativamente alta. A longa jornada de trabalho e a redução da qualidade de vida dos profissionais de enfermagem na UTI mostraram-se importantes fatores em comum para o desenvolvimento das DCNT.
\end{abstract}

Palavras-chave: Ambiente de trabalho; Enfermagem; Saúde do trabalhador; Doenças não transmissíveis.

\begin{abstract}
Objective: To describe the occurrence of self-reported Chronic Non-Communicable Diseases (NCDs) and related risk factors in nurses in the General and Pediatric Intensive Care Unit (ICU) in a reference hospital in the city of MaceióAL. Methods: Descriptive study, carried out from April to July 2019. Data were obtained through a questionnaire prepared by the authors, with questions regarding personal and sociodemographic data, occupational and life habits and whether they already had such habits before the work in the ICU, in addition to self-reported anthropometric data. Results: 16 nurses were interviewed, $87.5 \%$ female, aged between 25 and 50 years, who worked in the sector between 11 and 15 years. Regarding the Body Mass Index, $37.5 \%$ were overweight and $6.25 \%$ were obese. $12.5 \%$ of the nurses declared they were smokers and $43.75 \%$ of the interviewees reported having acquired some NCDs during their professional performance in the ICU. Conclusion: The occurrence of identified NCDs and risk factors for its development was relatively high. The long working hours and the reduction in the quality of life of nursing professionals in the ICU proved to be important factors in common for the development of NCDs.
\end{abstract}

Keywords: Working environment; Nursing; Occupational health; Noncommunicable diseases.

\section{Resumen}

Objetivo: Describir la ocurrencia de enfermedades crónicas no transmisibles (ENT) autodeclaradas y factores de riesgo relacionados en enfermeras de la Unidad de Cuidados Intensivos General y Pediátrica (UCI) de un hospital de 
referencia de la ciudad de Maceió-AL. Métodos: Estudio descriptivo cuantitativo, realizado de abril a julio de 2019.Los datos se obtuvieron a través de un cuestionario elaborado por los autores, con preguntas sobre datos personales y sociodemográficos, hábitos ocupacionales y de vida y si ya los tenían antes del trabajo en la UCI, además de los datos antropométricos autoinformados. Resultados: se entrevistaron 16 enfermeras, 87,5\% mujeres, con edades entre 25 y 50 años, que laboraron en el sector entre 11 y 15 años. En cuanto al Índice de Masa Corporal, el 37,5\% tenía sobrepeso y el 6,25\% obesidad. El 12,5\% de los enfermeros declaró ser fumador y el 43,75\% de los entrevistados refirió haber adquirido alguna ECNT durante su desempeño profesional en la UCI. Conclusión: La ocurrencia de ENT identificadas y los factores de riesgo para su desarrollo fue relativamente alta. Las largas jornadas laborales y la reducción de la calidad de vida de los profesionales de enfermería en la UCI resultaron ser factores importantes en común para el desarrollo de las ECNT.

Palabras clave: Ambiente de trabajo; Enfermería; Salud del trabajador; Enfermedades no transmisibles.

\section{Introdução}

A Unidade de Terapia Intensiva (UTI) destina-se à assistência de pacientes criticamente doentes e de alto risco que exigem cuidados contínuos, especializados e complexos, realizados por uma equipe de saúde multiprofissional (Leite \& Vila, 2005). A enfermagem exerce um papel significativo no processo de cuidado e reabilitação do paciente, sendo responsável por 60\% das ações de saúde e prestação de assistência 24 horas por dia. Esse grupo profissional constitui o maior contingente da força de trabalho da área da saúde, desempenhando diversas funções e responsabilidades (Rocha \& Almeida, 2000).

Os enfermeiros apresentam inúmeros fatores que podem favorecer o desgaste da saúde física e mental e a perda da qualidade de vida, como a sobrecarga de trabalho, redução na autonomia, escassez de materiais, estresse, baixos salários e situações diárias de sofrimento, morte e dor (Ferreira et al., 2006; Pappa et al., 2020). Esses fatores relacionados à genética, sexo, faixa etária, bem como a hábitos e comportamentos de risco, a exemplo de alimentação inadequada, inatividade física, tabagismo e consumo de bebidas alcoólicas, podem provocar alterações fisiológicas, sociais e psíquicas aos trabalhadores. Além disso, podem influenciar no desenvolvimento e/ou agravamento de doenças crônicas não transmissíveis (DCNT), que podem incluir doenças cardiovasculares, neoplasias, doenças respiratórias, diabetes, obesidade e doenças osteomusculares (Malta \& Silva Júnior, 2013).

As DCNT também podem acarretar custos financeiros aos pacientes e suas famílias e, principalmente, ao Sistema Único de Saúde (SUS). A sociedade, as empresas e o governo também são atingidos indiretamente devido à redução da produtividade, diminuição da qualidade da assistência prestada, aposentadoria precoce e ao alto índice de absenteísmo (Malta \& Silva Júnior, 2013). Por isso, as DCNT são consideradas um grave problema de saúde pública.

Segundo a Organização Mundial da Saúde (OMS), essas doenças foram responsáveis por 71\% das mortes no mundo e por 74\% do total de mortes no Brasil em 2016 (WHO, 2018). Em Alagoas, dados do Departamento de Informática do SUS (DATASUS) indicam que 124,8 mil pessoas são portadoras de doenças crônicas graves no estado. Entretanto, é deficiente o número de pesquisas voltadas à prevalência de DCNT em profissionais enfermeiros principalmente na UTI (Teixeira \& Mantovani, 2009).

Diante dessa problemática, este estudo teve como objetivo descrever a ocorrência de DCNT autorreferidas e de fatores de risco em enfermeiros da UTI Geral e Pediátrica em um hospital de referência na cidade de Maceió - AL. Este trabalho possibilitará aos pesquisadores e enfermeiros desenvolverem medidas de prevenção de doenças e promoção à saúde, identificando possíveis dificuldades da assistência de enfermagem prestada por profissionais com DCNT.

\section{Metodologia}

Estudo descritivo. A pesquisa foi realizada nas UTIs Geral e Pediátrica de um hospital público de Alagoas, nos meses de abril a julho de 2019. Foram utilizados os seguintes critérios de inclusão: enfermeiros com idade entre 20 e 59 anos, que estavam trabalhando regularmente na instituição por um período superior a três meses, que tinham concluído o curso de 
bacharelado em Enfermagem, que aceitaram participar do estudo e que assinaram o Termo de Consentimento Livre e Esclarecido (TCLE). Foram excluídos os profissionais que estavam de férias, de licença maternidade ou licença médica.

Inicialmente foi identificado um total de 22 profissionais, desses, dois estavam de férias e dois estavam de licença médica. Dessa forma, 18 profissionais estavam presentes na escala, mas apenas 16 aceitaram participar da pesquisa. Os enfermeiros foram convidados pessoalmente no início do plantão a responder um questionário próprio, elaborado pelos autores, autoaplicável, com perguntas abertas e fechadas referentes à idade, sexo, estado civil, religião, doenças em curso, frequência e hábitos de lazer, tempo e trabalho em UTI, quantidade de vínculos empregatícios, horas trabalhadas, procedimentos de enfermagem não ergonômicos e fatores do ambiente de trabalho que poderiam contribuir para o desenvolvimento da doença.

Para a realização do cálculo de Índice de Massa Corporal (IMC), foram utilizados dados antropométricos autorreferidos (peso, altura e circunferência abdominal) aplicando a fórmula e classificação da World Health Organization (1995). Para realizar o cálculo do IMC, divide-se o peso pela altura elevada ao quadrado, resultados inferiores a 18,5 indicam baixo peso, resultados entre 18,5 e 24,9 indicam que o peso está dentro da faixa de normalidade, resultados entre 25 e 29,9 indicam sobrepeso e acima de 30 indica obesidade (WHO, 1995). Segundo a OMS, a medida ideal de Circunferência da Cintura em homens é menor ou igual a $94 \mathrm{~cm}$ e o valor limítrofe é menor ou igual a $102 \mathrm{~cm}$, em mulheres, o valor ideal é menor ou igual a $80 \mathrm{~cm}$ e o valor limítrofe é menor ou igual a $88 \mathrm{~cm}$ (WHO, 1998). Os participantes também foram questionados quanto aos hábitos de vida (realização de exercícios físicos, consumo de álcool e cigarro, tipo de alimentação) e se esses hábitos já existiam antes da atuação na UTI.

Os dados foram organizados com auxílio do programa Excel 2010 e a análise foi realizada através de estatística descritiva. A pesquisa foi submetida a um Comitê de Ética em Pesquisa, recebendo parecer favorável sob CAAE: 98871018.1.0000.5011 e obedeceu a todos os princípios éticos de acordo com a Resolução n ${ }^{\circ}$ 510/2016 do Conselho Nacional de Saúde.

\section{Resultados e Discussão}

\section{Características sociodemográficas}

Neste estudo, foram entrevistados 16 enfermeiros, cuja maioria $(87,5 \%, \mathrm{n}=14)$ era do sexo feminino, com idade entre 25 a 50 anos e que atuava no setor entre 11 a 15 anos $(68,75 \%, \mathrm{n}=11)$. A distribuição dos profissionais de enfermagem de acordo com o sexo, faixa etária, cor, religião, estado civil, tempo de atuação na UTI e número de vínculos empregatícios está representada na Tabela 1 . 
Tabela 1 - Características sociodemográficas dos enfermeiros da Unidade de Terapia Intensiva (UTI) Geral e Pediátrica de um hospital público de Alagoas, Brasil, 2019.

\begin{tabular}{|c|c|c|}
\hline Variáveis sociodemográficas & $\mathbf{n}$ & $\%$ \\
\hline \multicolumn{3}{|l|}{ Sexo } \\
\hline Feminino & 14 & 87,50 \\
\hline Masculino & 2 & 12,50 \\
\hline \multicolumn{3}{|l|}{ Faixa etária } \\
\hline 20 a 30 anos & 1 & 6,25 \\
\hline 31 a 40 anos & 2 & 12,50 \\
\hline 41 a 50 anos & 13 & 81,25 \\
\hline \multicolumn{3}{|l|}{ Cor } \\
\hline Branca & 3 & 18,75 \\
\hline Parda & 13 & 81,75 \\
\hline \multicolumn{3}{|l|}{ Religião } \\
\hline Espírita & 1 & 6,25 \\
\hline Protestante & 4 & 25,00 \\
\hline Católica & 11 & 68,75 \\
\hline \multicolumn{3}{|l|}{ Estado civil } \\
\hline Solteiro & 2 & 12,50 \\
\hline Casado & 14 & 87,50 \\
\hline \multicolumn{3}{|l|}{ Tempo de atuação na UTI } \\
\hline 1 a 10 anos & 3 & 18,75 \\
\hline 11 a 20 anos & 11 & 68,75 \\
\hline 21 a 30 anos & 2 & 12,50 \\
\hline \multicolumn{3}{|c|}{ Número de vínculos empregatícios } \\
\hline 1 vínculo & 3 & 18,75 \\
\hline 2 vínculos & 11 & 68,75 \\
\hline 3 vínculos & 2 & 12,50 \\
\hline
\end{tabular}

Fonte: Dados da pesquisa (2019).

A predominância no presente estudo de trabalhadores do sexo feminino está relacionada a uma característica histórica ligada à profissão. No Brasil, as mulheres compõem $85,1 \%$ das equipes de enfermagem, os homens $14,4 \%$, e $0,5 \%$ não responderam a esse questionamento na pesquisa. Apesar do predomínio feminino, a partir da década de 1990 ocorreu uma tendência de crescente presença masculina nas equipes (Machado et al., 2015). Os resultados obtidos corroboram com os encontrados na literatura, de que a enfermagem é exercida, em sua maioria, por mulheres (De Lima et al., 2013; Domingues et al., 2019).

Percebeu-se que $87,5 \%$ dos profissionais entrevistados eram casados. O fato de serem casados pode influenciar na necessidade de mais vínculos empregatícios para aumentar a renda da família a prover o sustento financeiro. Entretanto, o trabalho exaustivo sobrecarrega o profissional e reduz o tempo de descanso, das atividades de lazer e de convívio com a família (De Lima et al., 2013).

Por sua vez, o reduzido tempo livre para atividades, como lazer, autocuidado, cultura e melhor convivência familiar desencadeia angústia nos trabalhadores de enfermagem. Sabe-se também que a boa qualidade do ambiente laboral está relacionada ao estabelecimento de relações familiares positivas e na evolução saudável socioemocional e educacional dos filhos. Na vida das pessoas, o trabalho pode influenciar nas condições físicas e mentais do trabalhador, afetando a relação de prazer e sofrimento (Silva et al., 2017).

O tempo de atuação em uma determinada função tem influência direta sobre a qualidade da atividade desempenhada. Grande parte dos pesquisados trabalhavam na UTI entre 11 e 20 anos. Tal resultado permite inferir que quanto mais experiência um trabalhador possui em uma determinada função e setor, maior é a responsabilidade atribuída a ele e maior é a cobrança por alta produtividade, constituindo uma circunstância agravante e que direciona ao estresse, ansiedade e adoecimento dos enfermeiros (Fontinhas \& Cardoso, 2017). O contato prolongado e contínuo com pacientes gravemente 
enfermos e o sentimento de alto grau de responsabilidade podem ocasionar consequências adversas, como patologias, absenteísmo, diminuição do desempenho, prejuízos emocionais, baixa produtividade e aumento do risco de ansiedade, que colocam em risco a vida dos profissionais e dos pacientes (Mokhtari et al., 2020). Além disso, a intensa pressão no ambiente de trabalho em UTI está relacionada à ocorrência de acidentes de trabalho (Ghorbanpoor et al., 2019).

Na presente pesquisa, foi observado que $81,25 \%$ dos profissionais tinham faixa etária entre 41-50 anos. Este resultado difere do observado em outro estudo em que a idade média dos profissionais de Enfermagem se encontrava entre 26-40 anos (Machado et al., 2015). É comprovado que o avanço da idade está relacionado ao desenvolvimento de DCNT em profissionais de Enfermagem, visto que, proporcionalmente ao avanço do envelhecimento da população, o número de mortes prematuras diminui e aumenta-se a ocorrência de DCNT devido à exposição de forma mais prolongada aos fatores de risco (Achutti \& Azambuja, 2004).

\section{Fatores de risco identificados para DCNT}

Em relação à alimentação, 12 profissionais relataram que ingeriam de 1 a 3 porções de frutas e/ou legumes durante o dia, 3 trabalhadores ingeriam de 3 a 5 porções por dia e apenas 1 não ingeria nenhuma porção de frutas e/ou legumes por dia (Gráfico 1A); 68,25\% ( $n=11)$ dos enfermeiros costumavam ingerir essa quantidade antes de atuar na UTI e 31,25\% ( $n=5)$ reduziram a quantidade ingerida devido à falta de tempo de preparar as refeições, visto que passavam pouco tempo em suas residências.

Quanto à ingestão de massas, frituras e alimentos ultra processados, 10 profissionais disseram que consumiam de $1 \mathrm{a}$ 3 vezes por semana, 4 ingeriam 3 a 5 vezes por semana, 1 trabalhador ingeria 5 a 8 vezes por semana e 1 relatou que não ingeria esses alimentos nenhuma vez (Gráfico 1B). Uma pesquisa realizada na Nigéria, entre 2016 e 2017, com 196 enfermeiros, mostrou que 23,5\% consumiam frituras de 3 a 7 vezes ao longo da semana e 91,8\% mantinham uma dieta não saudável (Buremoh et al., 2020).

O Guia Alimentar para a População Brasileira recomenda preferir sempre alimentos naturais, frescos ou minimamente processados à alimentos ultraprocessados e a diminuição do consumo de frituras e carnes vermelhas (Brasil, 2014). 
Gráfico 1 - Percentual do consumo de frutas e/ ou legumes por dia (em A) e do consumo de massas, frituras e alimentos ultra processados (em B) dos enfermeiros das Unidades de Terapia Intensiva (UTI) Geral e Pediátrica de um hospital público de Alagoas, Brasil, 2019.

A

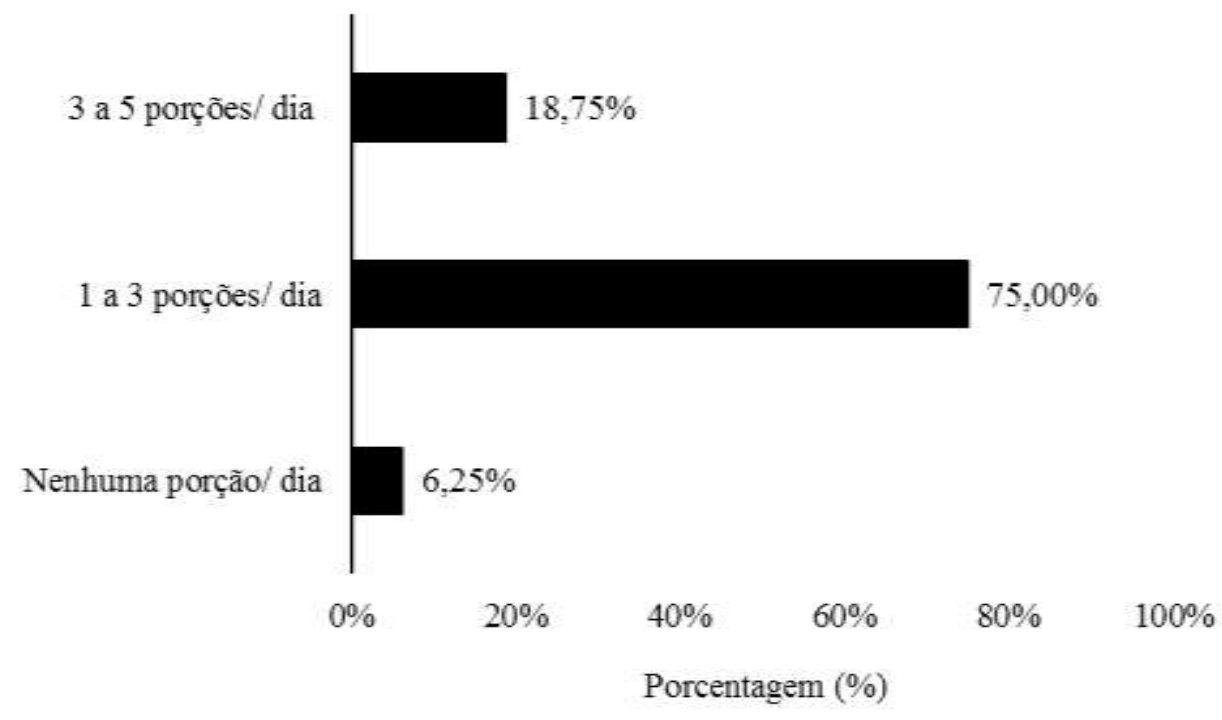

$\mathbf{B}$

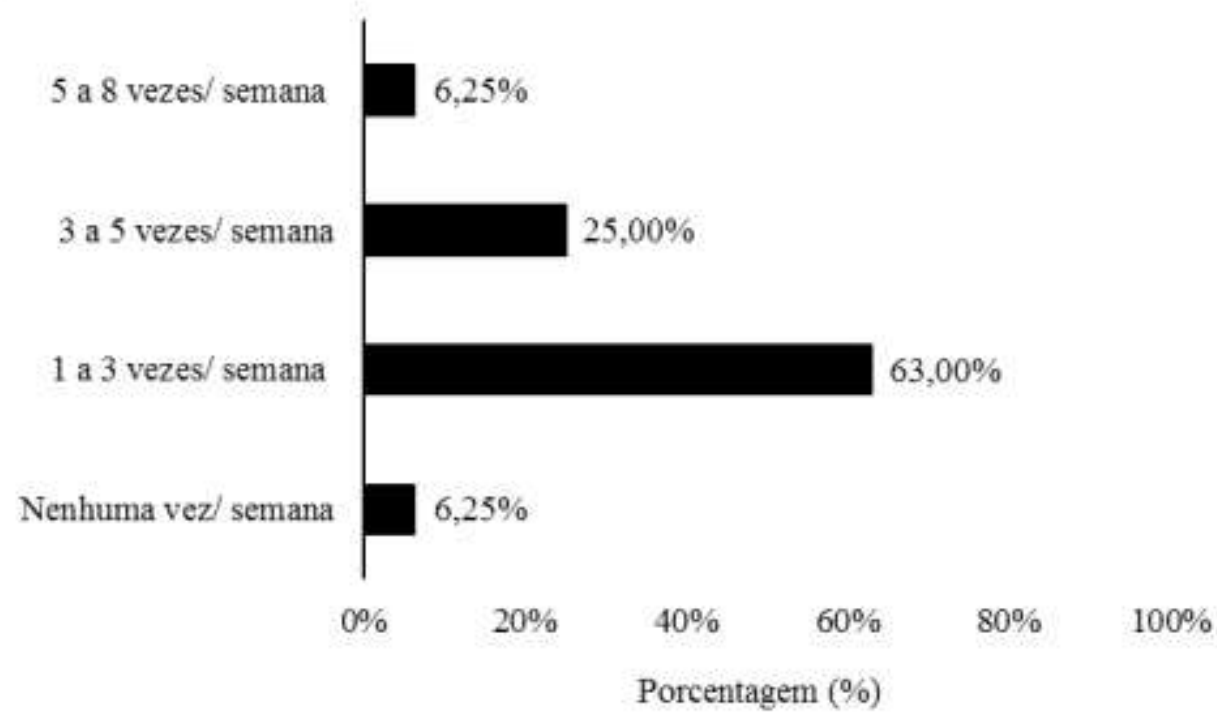

Fonte: Dados da pesquisa (2019).

O estilo de vida é um dos responsáveis pela alta prevalência de doenças crônicas na sociedade, especialmente atitudes que favorecem o aumento de peso corporal e a obesidade visceral, bem como o consumo rotineiro de alimentos industrializados. Além disso, a alimentação está diretamente relacionada a fatores que interferem na prevenção e no controle das doenças crônicas e seus agravos, como excesso de peso, dislipidemia, mau controle glicêmico e padrão alimentar com consumo excessivo de gordura saturada e pouca ingestão de frutas e vegetais (Brasil, 2014).

Em função das características enzimáticas e antioxidantes das vitaminas e minerais presentes em alimentos naturais, o seu consumo rotineiro é um fator de proteção contra as DCNT. Estudos demonstram que o consumo adequado desses 
alimentos está associado a um menor índice de mortalidade por doença cardiovascular e neoplasias (Wang et al., 2014). É recomendado pela OMS a ingestão de 400 gramas de frutas, legumes e hortaliças ao longo do dia, o que corresponde a cerca de cinco porções diárias (WHO, 2003).

Dentre as possíveis barreiras para o consumo moderado ( 1 a 3 vezes por dia) de frutas/ legumes pelos enfermeiros da UTI, estão: o custo/ preço desses alimentos; hábito/costume pessoal de consumir frutas/ legumes; falta de tempo para ir ao mercado/feira; falta de tempo para consumir no trabalho. Por outro lado, pode-se afirmar que o consumo de massas, panificados e embutidos se deve à conveniência do preparo, praticidade, palatividade e facilidade de acesso, associados à escassez do tempo e ao modo de vida das famílias modernas (Santos, 2019).

Além disso, a ingestão de carboidratos simples e gorduras saturadas de forma crônica tem sido associada ao aumento de risco de doenças cardiovasculares, devido ao aumento de peso corporal, aumento da pressão arterial, hiperlipidemia, hipertrofia ventricular esquerda e fibrose, intolerância à glicose, inflamação e esteatose hepática com comprometimento da atividade da cadeia respiratória mitocondrial (Alam et al., 2013).

Sobre a prática de atividade física, $56,25 \%(\mathrm{n}=9)$ dos enfermeiros afirmaram que realizavam algum exercício físico (exercício aeróbico e/ou musculação) com regularidade, 6 deles com a frequência de 1 a 3 vezes por semana, 2 com a frequência de 3 a 5 vezes por semana e 1 com a frequência de 5 a 7 vezes por semana. Os 7 (43,75\%) que não praticavam nenhum exercício físico relataram como justificativa o cansaço $(12,5 \%, \mathrm{n}=2)$, a redução de tempo disponível $(18,75 \%, \mathrm{n}=3)$ e a necessidade de passar mais tempo com a família $(12,5 \%, \mathrm{n}=2)$ (Gráfico $2 \mathrm{~A})$. Nove trabalhadores apresentaram o IMC dentro da faixa de normalidade, 6 apresentaram sobrepeso e 1 obesidade grau I (Gráfico 2B). 
Gráfico 2 - Percentual da prática de exercício físico (em A) e do Índice de Massa Corporal (IMC) (em B) dos enfermeiros das Unidades de Terapia Intensiva (UTI) Geral e Pediátrica de um hospital público Alagoas, Brasil, 2019.

A

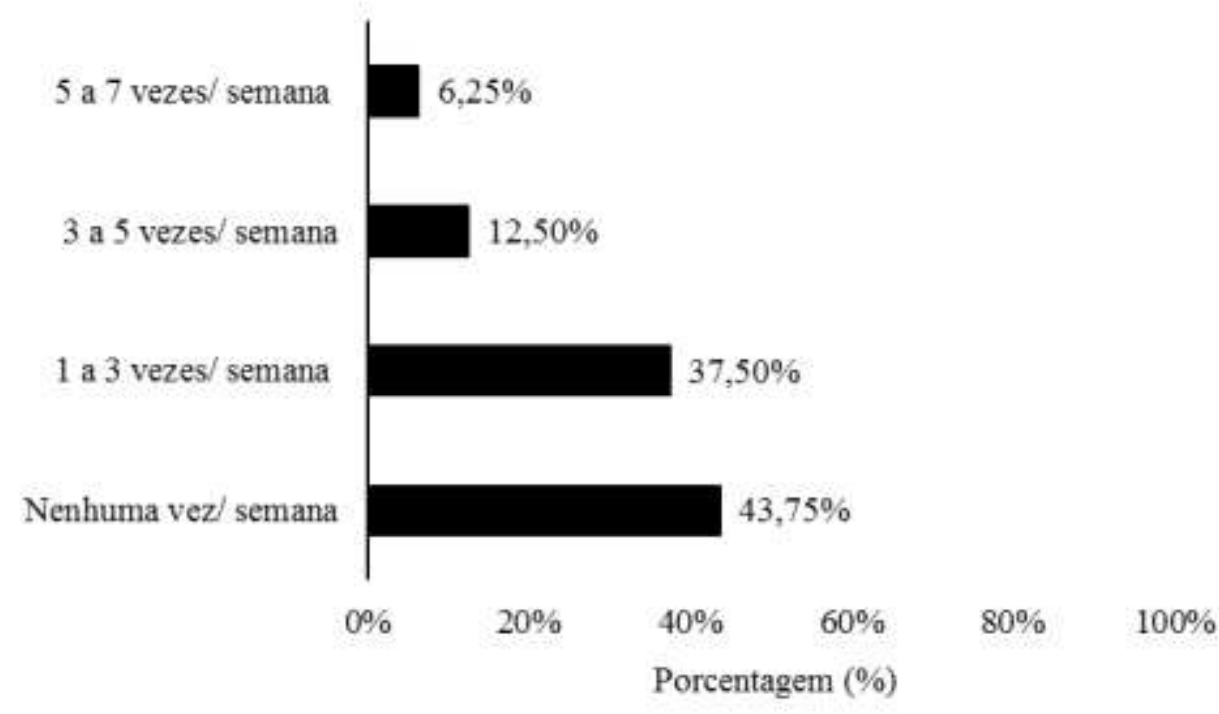

B

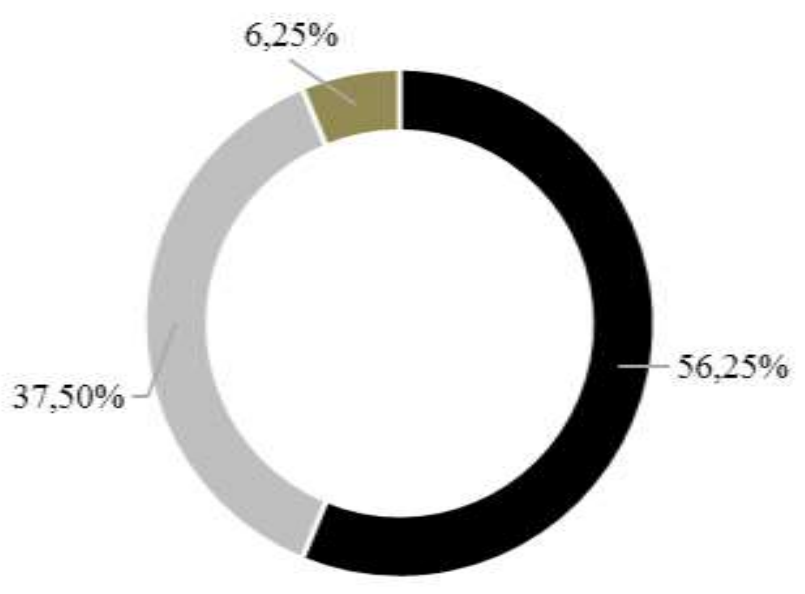

- Normal = Sobrepeso = Obesidade Grau I

Fonte: Dados da pesquisa (2019).

A OMS recomenda 150 minutos de atividade física moderada ou 75 minutos de atividade física intensa por semana (WHO, 2010). Sabe-se que a prática de exercícios físicos de forma regular pode minimizar, de forma significativa, o risco de mortalidade decorrente de problemas cardiovasculares em até 60\% (Tan et al., 2012). Acioli et al. (2013) e Freire et al. (2015) observaram que profissionais da UTI que praticavam atividade física possuíam maior qualidade de vida e saúde física. O aumento de massa muscular pode melhorar a imunidade, a disposição, o desempenho de atividades diárias, bem como prevenir lesões articulares e provocar mudanças positivas na qualidade do sono e no humor.

Ademais, os resultados da presente pesquisa se assemelham a um estudo realizado na região Sul do Brasil, composto por 185 profissionais de enfermagem, em que 54,6\% afirmaram que praticavam alguma atividade física (Scholze et al., 2017). 
Entretanto, na Nigéria, outros autores observaram que 77\% dos entrevistados eram sedentários (Buremoh et al., 2020).

Em relação ao IMC, a obesidade e o sobrepeso estão interligados ao aumento da morbimortalidade, porque propicia o surgimento de comorbidades, como diabetes, hipertensão, dislipidemias e síndrome metabólica, levando a complicações cardíacas e cardiovasculares (D O’Brien et al., 2014).

Um estudo realizado em um hospital no estado de São Paulo - Brasil, com 326 trabalhadores de enfermagem, mostrou que a maioria $(66,7 \%)$ dos voluntários eram sedentários e $60 \%$ estavam acima do peso; destes, $69(21,1 \%)$ eram obesos (Pereira et al., 2019). Buremoh et al., (2020) observaram que 40,8\% apresentaram IMC compatível com sobrepeso ou obesidade e 58,2\% apresentavam a medida da circunferência da cintura acima do adequado.

No que se refere à medida da cintura, segundo a V Diretriz Brasileira de Hipertensão Arterial, o limite de normalidade para circunferência da cintura é de $88 \mathrm{~cm}$ para mulheres e $102 \mathrm{~cm}$ para homens (Sociedade Brasileira de Cardiologia, 2007). Entretanto, no presente estudo, 7 (43,75\%) enfermeiras referiram ter mais de $88 \mathrm{~cm}$. No Rio Grande do Sul - Brasil, um estudo com 80 trabalhadores de enfermagem identificou que $75 \%$ deles estavam com a circunferência da cintura acima dos valores limítrofes (Botolli et al., 2009).

Com relação ao tabagismo e etilismo, $12,5 \%(\mathrm{n}=2)$ dos enfermeiros declararam ser fumantes. Um deles fumava cerca de 20 cigarros por dia, enquanto o outro fumava aproximadamente 60 cigarros por dia e 37,5\% ( $\mathrm{n}=6)$ consumiam bebidas alcoólicas de 1 a 2 vezes por semana. Considerado um dos maiores fatores de risco para as doenças cardiovasculares, o tabagismo é a maior causa de doença coronariana, tanto em homens quanto em mulheres, assim como já está comprovada a sua correlação com a doença cerebrovascular. Aqueles que fumam mais de 20 cigarros por semana aumentam em cinco vezes o risco de morte súbita. Assim como as doenças cardiovasculares e suas repercussões são a causa mais comum de morte entre os tabagistas (Brasil, 2014).

Na região Sul do Brasil, foi realizada uma pesquisa com 185 profissionais de enfermagem na qual se revelou que 12\% deles havia feito uso de tabaco e $61 \%$ havia consumido álcool nos últimos 3 meses. Esse mesmo estudo concluiu que, quanto mais desfavorável o ambiente de trabalho do enfermeiro, sobretudo na relação com médicos, suporte organizacional e autonomia, maior era o consumo de substâncias psicoativas (Scholze et al., 2017). E, o estudo de Machado et al. (2016) mostrou que as longas jornadas laborais, o trabalho noturno e a elevada carga horária semanal têm relação positiva com o consumo de álcool.

Ainda não foi comprovada a relação entre o consumo de bebidas alcoólicas e o risco para doenças crônicas em baixos níveis de consumo, entretanto, a partir de duas doses (30g) de destilados, duas latas de cerveja ou dois copos de vinho, foi observado um aumento da pressão arterial em homens e mulheres (Brasil, 2014).

Sabe-se que o lazer, além de ser considerado um ponto importante para a saúde mental e influencia positivamente na manutenção de uma vida saudável (WERLE, 2018). E a redução do tempo de lazer decorrente da excessiva carga horária de trabalho influencia na sobrecarga mental dos profissionais enfermeiros (Leite et al., 2017).

Entre os trabalhadores entrevistados, $62,25 \%(\mathrm{n}=10)$ afirmaram perceber uma redução na quantidade e qualidade de horas de lazer após o início da atuação no setor, devido à redução de tempo disponível e ao cansaço decorrente do trabalho, enquanto 37,5\% $(n=6)$ não perceberam diferença. Os profissionais mencionaram que, nas horas de lazer, costumavam fazer mais de uma atividade, o que se pode observar no Gráfico 3. Apenas 1 entrevistado marcou a opção "desejo não responder".

Quanto à ergonomia, 93,75\% $(\mathrm{n}=15)$ dos enfermeiros consideraram que as posturas adotadas durante os procedimentos de enfermagem não eram ergonômicas, dentre estes, $62,5 \%(\mathrm{n}=10)$ informaram que "sempre" adotaram essas posturas, enquanto $31,25 \%(n=5)$ comunicaram que adotavam essas posturas "às vezes". Os principais procedimentos descritos como relacionados à adoção de posições não ergonômicas estão representados no Gráfico 4. 
Gráfico 3 - Número de vezes em que as atividades realizadas nas horas de lazer foram citadas pelos enfermeiros das Unidades de Terapia Intensiva (UTI) Geral e Pediátrica de um hospital público de Alagoas, Brasil, 2019.

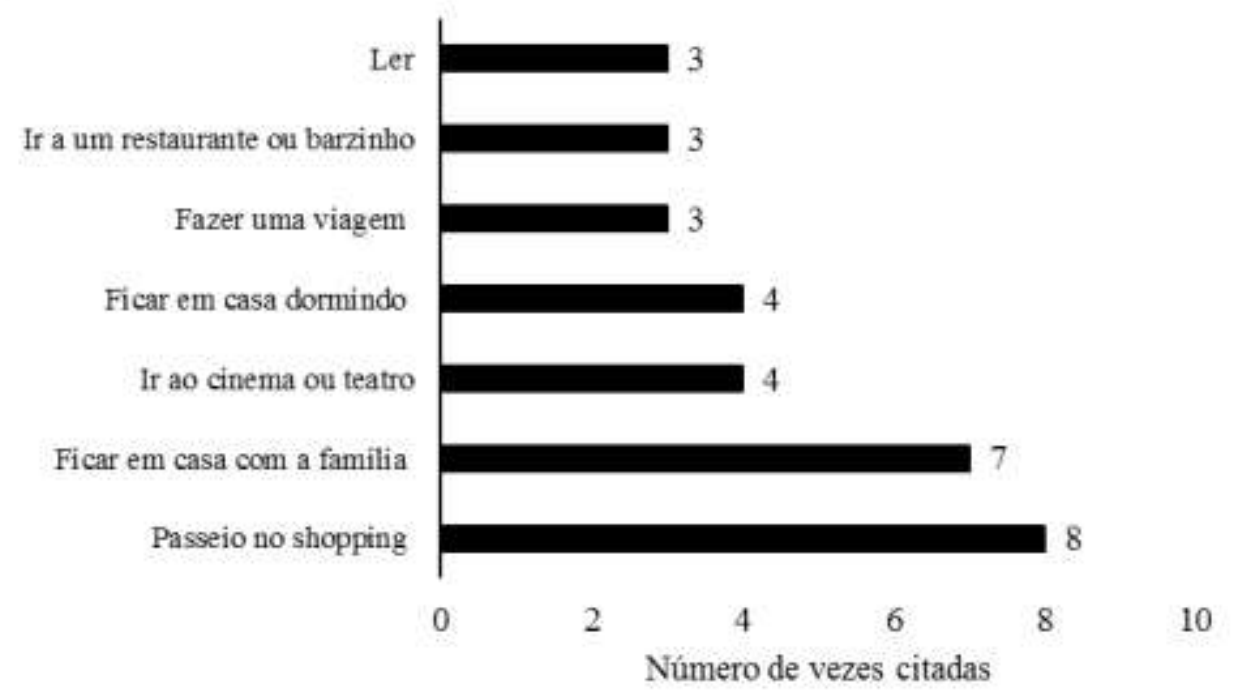

Fonte: Dados da pesquisa (2019).

Gráfico 4 - Frequência em que as atividades realizadas associadas a adoção de posturas não ergonômicas foram citadas pelos enfermeiros da Unidade de Terapia Intensiva (UTI) Geral e Pediátrica de um hospital público de Alagoas, Brasil, 2019.

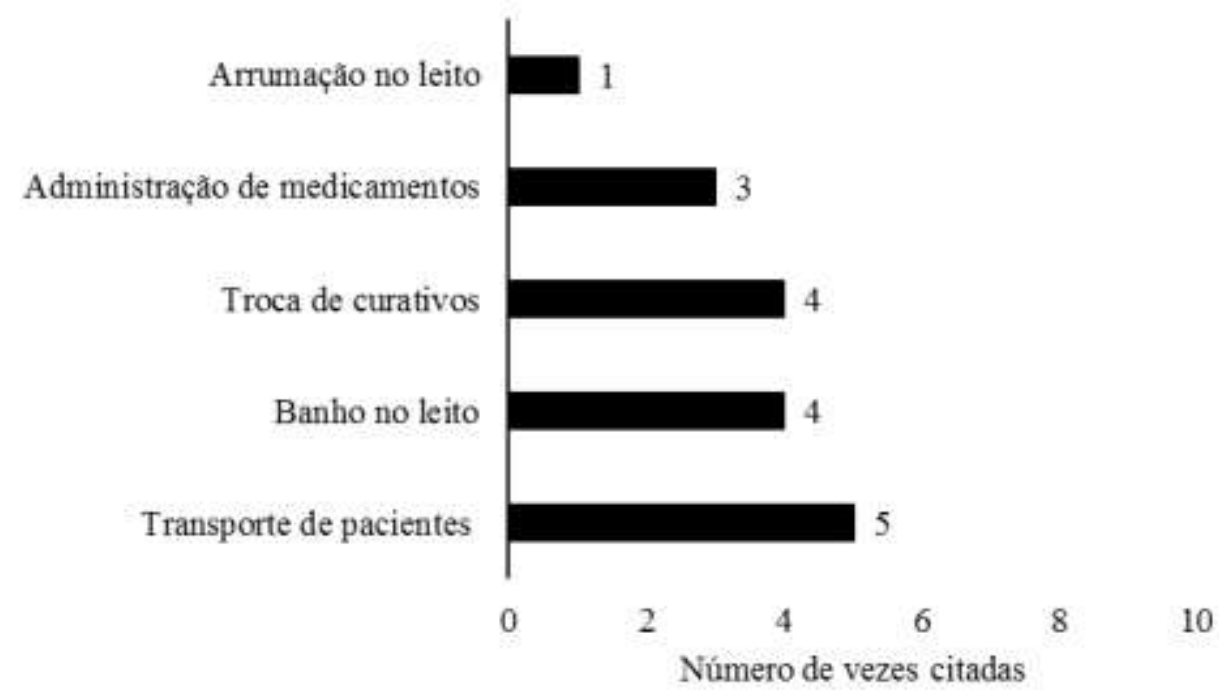

Fonte: Dados da pesquisa (2019).

Quando questionados sobre os fatores no ambiente de trabalho que poderiam contribuir para a progressão da doença, os profissionais indicaram mais de uma vez: leitos dos pacientes inapropriados para o manuseio ergonômico do profissional e falta de insumos, como medicamentos e materiais para realizar procedimentos básicos. Foram mencionadas 2 vezes estresse e ansiedade, enquanto que o problema de cadeiras em quantidade insuficiente para a equipe sentar-se ao longo do plantão foi citado 3 vezes e a sobrecarga de responsabilidades devido ao subdimensionamento da equipe de enfermagem, 4 vezes.

Segundo um estudo, profissionais de enfermagem em UTIs realizam várias atividades que exigem esforço físico, como manusear o paciente e os monitores, organizar os equipamentos, solicitar, organizar e preparar materiais de consumo no 
posto de trabalho e separar os equipamentos e móveis com problemas para reparos. Ao realizar essas atividades, os profissionais adotam posturas não ergonômicas e realizam movimentos mecânicos e repetitivos que contribuem para o desenvolvimento de algumas doenças crônicas, como Lesões por Esforço Repetitivo e Distúrbios Osteomusculares Relacionados ao Trabalho (LER/DORT), lombalgia, artralgia e hérnia de disco (Nishide \& Benatti, 2004). Ressalta-se que a perda de massa óssea durante o climatério, decorrente da queda nos níveis de estrogênio e de outros fatores associados, contribui para a fragilidade óssea (SOBRAC, 2003).

Conforme verificado neste trabalho, $93,75 \%$ dos enfermeiros consideraram que as posturas adotadas durante os procedimentos de enfermagem não eram ergonômicas. Em uma pesquisa realizada na Austrália, lesões musculoesqueléticas foram comumente referidas e quase $20 \%$ dos enfermeiros relataram problemas que restringiram atividades, indicando consequências adversas das atividades ocupacionais e domésticas (Reed et al., 2014).

Um estudo realizado na Turquia, com 102 profissionais de UTI, revelou que mais da metade dos trabalhadores apresentaram dores no pescoço, costas e punho e enfermeiros de UTI apresentaram riscos ergonômicos elevados (Dilek et al., 2018). Uma outra pesquisa realizada em 2019, na China, com 679 enfermeiros, mostrou que 97\% dos entrevistados relataram ter experimentado pelo menos um distúrbio musculoesquelético relacionado ao trabalho no ano anterior. A lombalgia foi o distúrbio musculoesquelético mais comumente relatado $(80,1 \%)$, sucedido pela cervicalgia $(78,6 \%)$ e dores no ombro $(70,4 \%)$ (Yang et al., 2019).

\section{Ocorrência de DCNT e associação com os fatores de risco}

Dentre os entrevistados, $81,25 \%(n=13)$ relataram ter histórico familiar (genitores) de alguma DCNT e 43,75\% ( $n=$ 7) disseram ter desenvolvido alguma DCNT ao longo da atuação profissional na UTI, diagnosticada há 6,2 anos em média. Dentre os 7 enfermeiros que desenvolveram alguma DCNT, $5(31,25 \%)$ apresentaram mais de uma concomitantemente. A Tabela 2 relaciona as DCNT com os fatores de risco investigados. Pode-se observar no Quadro 1 que existe uma possível relação entre as longas jornadas de trabalho, a idade das profissionais, a inatividade física, a alimentação não adequada, bem como as posturas não ergonômicas, e o desenvolvimento das DCNT.

As extensas e exaustivas jornadas de trabalho contribuem para a diminuição do autocuidado dos profissionais de enfermagem, uma vez que restringem a sua disponibilidade para usufruir de atividades de lazer/ descanso, praticar atividades físicas, assim como de uma alimentação equilibrada. É importante ressaltar que estudos identificaram outros fatores favoráveis para o desencadeamento das DCNT, como: recursos inadequados, baixo poder de decisão, falta de reconhecimento de seu trabalho, remuneração insuficiente, sobrecarga de trabalho ao desempenhar vários papéis e funções, assim como o tempo e a qualidade do sono (Domingues et al., 2019). 
Research, Society and Development, v. 10, n. 10, e167101017523, 2021

(CC BY 4.0) | ISSN 2525-3409 | DOI: http://dx.doi.org/10.33448/rsd-v10i10.17523

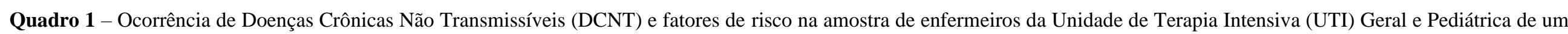
hospital público de Maceió-AL, 2019.

\begin{tabular}{|c|c|c|c|c|c|c|c|}
\hline $\begin{array}{c}\text { Fatores de risco/ } \\
\text { profissionais }\end{array}$ & Profissional 1 & Profissional 2 & Profissional 3 & Profissional 4 & Profissional 5 & Profissional 6 & Profissional 7 \\
\hline DCNT & $\begin{array}{c}\text { Hérnia de disco e } \\
\text { lombalgia }\end{array}$ & $\begin{array}{c}\text { Câncer de mama e } \\
\text { diabetes }\end{array}$ & $\begin{array}{c}\text { Dislipidemia e } \\
\text { artralgia }\end{array}$ & Depressão & Artrose e lombalgia & Artralgia & $\begin{array}{c}\text { Hipertensão e } \\
\text { obesidade }\end{array}$ \\
\hline $\begin{array}{c}\text { Carga horária } \\
\text { semanal }\end{array}$ & 60 horas & 70 horas & 40 horas & 40 horas & 64 horas & 60 horas & 60 horas \\
\hline $\begin{array}{c}\text { Consumo de frutas e } \\
\text { verduras }\end{array}$ & $\begin{array}{c}1 \text { a } 3 \text { vezes de frutas e } \\
\text { verduras por } \\
\text { dia }\end{array}$ & 1 a 3 vezes por dia & 1 a 3 vezes por dia & 1 a 3 vezes por dia & 1 a 3 vezes por dia & 1 a 3 vezes por dia & 1 a 3 vezes por dia \\
\hline $\begin{array}{c}\text { Consumo de massas, } \\
\text { frituras e alimentos } \\
\text { processados }\end{array}$ & $\begin{array}{c}3 \text { a } 5 \text { vezes por } \\
\text { semana de massas } \\
\text { por semana }\end{array}$ & $\begin{array}{c}1 \text { a } 3 \text { vezes por } \\
\text { semana }\end{array}$ & $\begin{array}{c}3 \text { a } 5 \text { vezes por } \\
\text { semana de massas } \\
\text { por semana }\end{array}$ & $\begin{array}{c}3 \text { a } 5 \text { vezes por } \\
\text { semana de massas } \\
\text { por semana }\end{array}$ & $\begin{array}{l}1 \text { a } 3 \text { vezes por } \\
\text { semana }\end{array}$ & $\begin{array}{c}1 \text { a } 3 \text { vezes por } \\
\text { semana }\end{array}$ & $\begin{array}{c}\text { e } 5 \text { a } 8 \text { vezes por } \\
\text { semana }\end{array}$ \\
\hline Tabagismo & Não & $\begin{array}{l}\text { Sim }(20 \\
\text { cigarros) }\end{array}$ & Não & Não & Não & Não & Não \\
\hline $\begin{array}{c}\text { Consumo de } \\
\text { bebidas alcoólicas }\end{array}$ & Não & Não & Não & $\begin{array}{l}1 \text { a } 2 \text { vezes por } \\
\text { semana }\end{array}$ & $\begin{array}{l}1 \text { a } 2 \text { vezes por } \\
\text { semana }\end{array}$ & $\begin{array}{l}1 \text { a } 2 \text { vezes por } \\
\text { semana }\end{array}$ & Não \\
\hline Exercício físico & Não pratica & Não pratica & Não pratica & Não pratica & $\begin{array}{l}3 \text { a } 5 \text { vezes } \\
\text { por semana }\end{array}$ & $\begin{array}{l}1 \text { a } 3 \text { vezes } \\
\text { por semana }\end{array}$ & Não pratica \\
\hline IMC & Normal & Sobrepeso & Normal & Normal & Normal & Sobrepeso & Obesidade \\
\hline Antecedentes familiares & Mãe hipertensa & Mãe diabética & Pai diabético & Mãe hipertensa & $\begin{array}{c}\text { Pai e mãe hipertensos } \\
\mathrm{e} \\
\text { diabéticos }\end{array}$ & Mãe hipertensa & $\begin{array}{c}\text { Mãe hipertensa e } \\
\text { pai diabético }\end{array}$ \\
\hline $\begin{array}{c}\text { Atividades não } \\
\text { ergonômicas realizadas } \\
\text { na UTI }\end{array}$ & $\begin{array}{c}\text { Trocas de curativos e } \\
\text { administração de } \\
\text { medicamentos }\end{array}$ & $\begin{array}{l}\text { Transporte de } \\
\text { paciente }\end{array}$ & $\begin{array}{l}\text { Transporte de } \\
\text { paciente }\end{array}$ & Banho no leito & \begin{tabular}{|c|} 
Administração de \\
medicamentos e \\
trocas de curativos
\end{tabular} & Trocas de curativos & Banho no leito \\
\hline
\end{tabular}

Legenda: DCNT - Doenças Crônicas Não Transmissíveis; IMC - Índice de Massa Corporal; UTI - Unidade de Terapia Intensiva. Fonte: Dados da pesquisa (2019). 
Em 2017, foi realizada uma pesquisa com profissionais da equipe de enfermagem no estado do Rio Grande do Sul, Brasil, na qual foi possível observar que 30\% dos profissionais possuíam alguma DCNT, e as observadas com maior prevalência foram a hipertensão arterial (20,6\%), doenças respiratórias crônicas (6,3\%) e Diabetes mellitus (5,5\%) (Domingues et al., 2019). De forma contrária, na presente pesquisa as doenças musculoesqueléticas prevaleceram entre os pesquisados.

\section{Considerações Finais}

A ocorrência de enfermeiros que desenvolveram alguma DCNT ao longo da atuação na UTI apresentou-se relativamente alta, com $43,75 \%$ dos entrevistados. Entre os fatores de risco identificados, a longa jornada de trabalho mostrouse um importante fator em comum para o desenvolvimento das DCNT e redução da qualidade de vida do profissional de enfermagem na UTI. Assim, recomenda-se que sejam incorporadas à rotina hospitalar, medidas que ofereçam melhores condições de trabalho, como a prática frequente de ginástica laboral, o ajuste de funções e setores, o incentivo a mudanças no estilo de vida, consultas médicas periódicas, além da criação de programas educativos. Apesar do número amostral ser limitado, das medidas antropométricas autorreferidas apresentarem viés de mensuração e das respostas as perguntas relatarem hábitos de muitos anos antes, podendo refletir viés de esquecimento, dificultando a avaliação dos efeitos, foram observados resultados semelhantes em estudos prévios de natureza similar. Dessa forma, é necessária a realização de mais estudos sobre essa temática e a ampliação desta pesquisa a outras instituições de saúde.

\section{Agradecimentos}

Ao Programa Institucional de Bolsas de Iniciação Científica da Universidade Estadual de Ciências da Saúde de Alagoas / Fundação de Amparo à Pesquisa do Estado de Alagoas (PIBIC/FAPEAL) - 2019-2020.

\section{Referências}

Brasil (2013). Ministério da Saúde. Secretaria de Atenção à Saúde. Departamento de Atenção Básica. Estratégias para o cuidado da pessoa com doença crônica: diabetes mellitus. Ministério da Saúde, Secretaria de Atenção à Saúde, Departamento de Atenção Básica. Ministério da Saúde.

Brasil (2014). Ministério da Saúde. Secretaria de Atenção à Saúde. Departamento de Atenção Básica. Guia alimentar para a população brasileira. Ministério da Saúde, Secretaria de Atenção à Saúde, Departamento de Atenção Básica. Ministério da Saúde.

Buremoh, A. I., Akindele, A., \& Omokhodion, F. O. (2020). Lifestyle habits and cardiovascular risk factors among urses at The University College Hospital, Ibadan, Southwest Nigeria. African Journal of Biomedical Research, 23(SE1), 111-118.

D O’Brien, P., Hinder, L. M., Callaghan, B. C., \& Feldman, E. L. (2017). Neurological consequences of obesity. The Lancet Neurology, $16(6)$, 465-477.

Da Silva, J. O., de Almeida Ferreira, S. K., Silva, S. F., Bergamini, G. B., Samuelsson, E., Joner, C., Schneider, L. F., \& Menz, P. R. (2017). A correlação existente entre o estresse no ambiente de trabalho e doenças psicossomáticas. Revista Científica da Faculdade de Educação e Meio Ambiente, 8(2), 177-191.

Da Silva Machado, A., Monteiro, P. S., Ribeiro, L. M., \& Guilhem, D. (2016). Consumo de alcool por enfermeiros e implicações para o trabalho em saúde: Revisão integrativa. Cogitare Enferm, 21(4), 1-8.

De Campos Teixeira, R., \& de Fátima Mantovani, M. (2009). Enfermeiros com doença crônica: As relações com o adoecimento, a prevenção e o processo de trabalho. Revista da Escola de Enfermagem da USP, 43(2), 415-421.

De Lima, M. B., Da Silva, L. M. S., Almeida, F. C. M., Torres, R. A. M., \& Dourado, H. H. M. (2013). Agentes estressores em trabalhadores de enfermagem com dupla ou mais jornada de trabalho. Revista de Pesquisa Cuidado é Fundamental Online, 5(1), 3259-3266.

Dilek, B., Beyan, A. C., Özcan, S., Demirel, T., Işık, Ö., \& Demiral, Y. (2018). 27 Ergonomic risk factors in intensive care unit and musculoskeletal symptoms. BMJ Publishing Group Ltd.

Domingues, J. G., Silva, B. B. C. da, Bierhals, I. O., \& Barros, F. C. (2019). Doenças crônicas não transmissíveis em profissionais de enfermagem de um hospital filantrópico no Sul do Brasil. Epidemiologia e Serviços de Saúde, 28, e2018298.

Ferreira, S. M., Rodrigues, E. A. D. O. N., da silva, A. M., de Oliveira, A. S., \& da Silva, A. C. (2006). A exaustão do profissional de enfermagem relacionado às condições de trabalho. Revista Uningá, 10(1).

Fontinhas, J. E., \& Cardoso, J. M. M. (2017). O estresse no trabalho do enfermeiro. Revista Uningá, 51(1). 
Freire, C. B., Dias, R. F., Schwingel, P. A., França, E. E. T. de, Andrade, F. M. D. de, Costa, E. C., \& Correia Junior, M. A. V. (2015). Qualidade de vida e atividade física em profissionais de terapia intensiva do sub médio São Francisco. Rev Bras Enferm, 68(1), 26-31.

Ghorbanpoor, F., Jouybari, L., Sanagoo, A., \& Pahlavanzadeh, B. The relationship between perceived occupational pressure with occupational accidents among nurses in ICU. Journal of Nursing Education, 8(1), 2019.

Leite, M. A., \& Vila, V. da S. C. (2005). Dificuldades vivenciadas pela equipe multiprofissional na unidade de terapia intensiva. Revista Latino-Americana de Enfermagem, 13(2), 145-150.

Leite, B. B., Barros, L M P de, Soares, P. R; Bento, T. M. A. \& Silva, L. K. B. da. (2017). Fatores associados à depressão no profissional enfermeiro. Trabalho de Conclusão de Curso. Centro Universitário Tiradentes, Maceió, AL, Brasil.

Machado, M. H., Aguiar Filho, W., de Lacerda, W. F., de Oliveira, E., Lemos, W., Wermelinger, M., Vieira, M., dos Santos, M. R., de Sousa Junior, P. B., Justino, E., \& Barbosa, C. (2015) Características gerais da enfermagem: o perfil sócio demográfico. Enferm. Foco, 6(7,) p. 11-17.

Malta, D. C., \& Silva Jr, J. B. da. (2013). O Plano de ações estratégicas para o enfrentamento das doenças crônicas não transmissíveis no Brasil e a definição das metas globais para o enfrentamento dessas doenças até 2025: Uma revisão. Epidemiologia e Serviços de Saúde, 22(1), 151-164.

Mokhtari, R., Moayedi, S., \& Golitaleb, M. (2020). COVID-19: Pandemic and health anxiety among nurses of intensive care units. International Journal of Mental Health Nursing, 29(6), 1275.

Neto, A. A., Araújo, R., Pitangui, A., Menezes, L., França, E., Costa, E., \&Junior, M. C. (2013). Qualidade de vida e nível de atividade física de profissionais de saúde de unidades de terapia intensiva. Revista Brasileira de Atividade Física \& Saúde, 18(6), 711.

Nishide, V. M., \& Benatti, M. C. C. (2004). Occupational risks among a nursing staff working in an intensive care unit. Revista da Escola de Enfermagem da USP, 38(4), 406-414.

Pappa, S., Ntella, V., Giannakas, T., Giannakoulis, V. G., Papoutsi, E., \& Katsaounou, P. (2020). Prevalence of depression, anxiety, and insomnia among healthcare workers during the COVID-19 pandemic: A systematic review and meta-analysis. Brain, Behavior, and Immunity. 88, $901-907$.

Pereira, R. S. F., de Gusmão, J. L., dos Santos, C. A., \& Silva, A. (2017). Obesidade e sobrepeso em trabalhadores da enfermagem de um hospital público em São José dos ampos-SP. Revista Enfermagem Atual In Derme, 82(20).

Reed, L. F., Battistutta, D., Young, J., \& Newman, B. (2014). Prevalence and risk factors for foot and ankle musculoskeletal disorders experienced by nurses. BMC Musculoskeletal Disorders, 15(1), 1-7.

Rocha, S. M. M., \& Almeida, M. C. P. de. (2000). O processo de trabalho da enfermagem em saúde coletiva e a interdisciplinaridade. Revista LatinoAmericana de Enfermagem, 8(6), 96-101.

Santos, G. M. G. C. dos, Silva, A. M. R., Carvalho, W. O. de, Rech, C. R., \& Loch, M. R. (2019). Barreiras percebidas para o consumo de frutas e de verduras ou legumes em adultos brasileiros. Ciência \& Saúde Coletiva, 24, 2461-2470.

Scholze, A. R., Martins, J. T., Galdino, M. J. Q., \& Ribeiro, R. P. (2017). Ambiente ocupacional e o consumo de substâncias psicoativas entre enfermeiros. Acta Paulista de Enfermagem, 30(4), 404-411.

Tan, S., Li, W., \& Wang, J. (2012). Effects of six months of combined aerobic and resistance training for elderly patients with a long history of type 2 diabetes. Journal of Sports Science \& Medicine, 11(3), 495.

SOBRAC - Sociedade Brasileira de Climatério (2003). Consenso Brasileiro Multidisciplinar de Assistência à Saúde da Mulher Climatérica. SOBRAC. Editora Segmento, p. 219-270.

Sociedade Brasileira de Cardiologia (2007). V diretrizes brasileiras de hipertensão arterial. Arquivos Brasileiros de Cardiologia, 89 (3), p.24-79.

Wang, X., Ouyang, Y., Liu, J., Zhu, M., Zhao, G., Bao, W., \& Hu, F. B. (2014). Fruit and vegetable consumption and mortality from all causes, cardiovascular disease, and cancer: Systematic review and dose-response meta-analysis of prospective cohort studies. Bmj, 349.

Werle, V. (2018). Relações entre lazer e saúde em tempos de cultura somática. Revista Brasileira de Estudos do Lazer, 5(2), 20-3.

WHO - World Health Organization (1995). Physical status: the use and interpretation of anthropometry. Technical Report Series 854. World Health Organization.

WHO - World Health Organization (1998). Obesity: preventing and managing the global epidemic. Technical Report Series 894. World Health Organization.

WHO - World Health Organization (2003). Diet, nutrition and the prevention chronic diseases. World Health Organization.

WHO - World Health Organization (2010). Global recommendations on physical activity for health. World Health Organization, 60.

WHO - World Health Organization (2018). World health statistics 2018: monitoring health for the SDGs, sustainable development goals. World Health Organization.

Yang, S., Lu, J., Zeng, J., Wang, L., \& Li, Y. (2019). Prevalence and risk factors of work-related musculoskeletal disorders among intensive care unit nurses in China. Workplace Health \& Safety, 67(6), 275-287. 